\title{
Facial fear processing and psychotic symptoms in schizophrenia: functional magnetic resonance imaging study
}

Panayiota G. Michalopoulou, Simon Surguladze, Lucy A. Morley, Vincent P. Giampietro, Robin M. Murray and Sukhwinder S. Shergill

\section{Background}

The recognition of negative facial affect is impaired in people with schizophrenia. The neural underpinnings of this deficit and its relationship to the symptoms of psychosis are still unclear.

\section{Aims \\ To examine the association between positive and negative psychotic symptoms and activation within the amygdala and extrastriate visual regions of patients with schizophrenia during fearful and neutral facial expression processing. \\ Method \\ Functional magnetic resonance imaging was used to measure neural responses to neutral and fearful facial expressions in 11 patients with schizophrenia and 9 healthy volunteers during an implicit emotional task.}

\section{Results}

No association between amygdala activation and positive symptoms was found; the activation within the left superior temporal gyrus was negatively associated with the negative symptoms of the patients.

\section{Conclusions}

Our results indicate an association between impaired extrastriate visual processing of facial fear and negative symptoms, which may underlie the previously reported difficulties of patients with negative symptoms in the recognition of facial fear.

\section{Declaration of interest}

None.
People with schizophrenia display pronounced difficulties in recognising negative facial affect, such as fearful expression. ${ }^{1,2}$ The neural basis of this deficit and its relationship to symptoms of psychosis are still unclear, although correlations with both positive and negative psychotic symptoms have been reported. ${ }^{3,4}$ Visual perception of facial fear in healthy individuals involves face-responsive visual regions, i.e. the fusiform gyrus and the cortex surrounding the superior temporal sulcus as well as the amygdala. ${ }^{5,6}$ We used functional magnetic resonance imaging (fMRI) to examine the hypothesis that activation within these regions would be restricted in people with schizophrenia during processing of facial fear. More specifically, as amygdala activation has been associated with emotional salience and the formation of positive psychotic symptoms, ${ }^{7}$ we predicted that the participants with schizophrenia relative response in the amygdala would demonstrate a positive relationship with positive psychotic symptoms. As visual cortical perception deficits in patients with schizophrenia have been associated with negative symptoms, ${ }^{8,9}$ we also predicted that activation within the extrastriate visual regions would correlate negatively with the severity of negative symptoms in the participants with schizophrenia.

\section{Method}

Eleven people (all dextral $)^{10}$ with a DSM-IV diagnosis of schizophrenia ${ }^{11}$ were recruited from the Maudsley Hospital, London. All of these individuals, of whom nine were men and two were women, were receiving out-patient treatment with stable dosages of antipsychotic medication at the time of the study, with a chlorpromazine equivalent daily dosage of $523 \mathrm{mg}$ (s.d. $=455$, range 166-1666); eight patients were treated with conventional and three with atypical antipsychotics. The severity of negative and positive symptoms of the patients was assessed with the Positive and Negative Syndrome Scale (PANSS). ${ }^{12}$ All the PANSS ratings were completed by L.A.M. after appropriate training.

Nine healthy dextral individuals (five men, four women) comparable for age and years of education were recruited as a comparison group from the local population. Exclusion criteria for all participants were illicit substance or alcohol misuse within the past 2 years, a history of neurological illness, head injury or other significant medical illness. All participants had normal premorbid IQ scores, estimated with the National Adult Reading Test ${ }^{13}$ control group participants had significantly higher scores $(t=3.49$, d.f. $=18$, two-tailed $P=0.003)$. Written informed consent was obtained for all participants and the study was approved by the Institute of Psychiatry research ethics committee.

\section{Imaging study}

\section{Procedure}

The participants took part in an event-related fMRI experiment while viewing grey-scale images depicting prototypical facial expressions of fear and sadness, a neutral facial expression and a fixation cross. The facial stimuli were from the standard set of prototypical facial expressions of the six basic emotions by Ekman \& Friesen. ${ }^{14}$ The 'sad face' processing data are not discussed in this paper.

The sequence of the four stimuli (fearful face/sad face/neutral face/fixation cross) was randomised and common to all participants. Twenty stimuli were presented per condition, depicting either a face or the fixation cross, each presented for $3 \mathrm{~s}$ with an inter-stimulus interval of $6 \mathrm{~s}$, and the duration of the experiment was $8 \mathrm{~min}$. This was an implicit emotional processing task, with participants indicating the gender of the face by moving a joystick. No response was required to the fixation cross. 


\section{Data acquisition and image analysis}

Data were acquired using a $1.5 \mathrm{~T}$ scanner at the Maudsley Hospital, London, and analysed with software developed at the Institute of Psychiatry, London, using a standard non-parametric approach. Whole-brain analysis of variance was used to estimate significant within-group and between-group effects. A detailed description of the data acquisition and image analysis is available as a data supplement to the online version of this paper. A correlational analysis was used to examine associations between PANSS positive and negative sub-scale scores and activation within the brain regions specified in our a priori hypotheses: the fusiform gyrus, the superior temporal gyrus and the amygdala.

\section{Results}

The demographic and clinical characteristics of the participants are shown in Table 1.

\section{Behavioural results}

There was no significant difference in performance between the patient and control groups in the gender discrimination task (Mann-Whitney $U=38.0, z=-0.90$, two-tailed $P=0.37$ for fearful expression; Mann-Whitney $U=37.5, z=-0.94, P=0.35$ for neutral facial expression).

\section{Imaging results}

Within-group comparisons

The whole-brain analysis of variance (ANOVA) revealed the following within-group significant differences:

Control group: fearful v. neutral faces. In the control group the processing of fearful faces elicited significantly greater activation within the bilateral amygdala, right-sided parahippocampal gyrus, fusiform gyrus, superior temporal gyrus, middle occipital and temporal gyri and right insula than did processing of neutral faces (Table 2; Figs 1(a), 2(a)).

\begin{tabular}{|c|c|c|c|c|}
\hline & \multicolumn{2}{|c|}{ Schizophrenia group $(n=11)$} & \multicolumn{2}{|c|}{ Control group $(n=9)$} \\
\hline & Mean (s.d.) & Range & Mean (s.d.) & Range \\
\hline Age, years & $35(9)$ & $20-53$ & $32(6)$ & $25-45$ \\
\hline NART IQ & $106.18(10.41)$ & 87-123 & $119.44(5.03)$ & $111-125$ \\
\hline Education, years & $13(2)$ & $10-16$ & $15(3)$ & $10-19$ \\
\hline Duration of illness, years & $12(9)$ & $2-33$ & & \\
\hline \multicolumn{5}{|l|}{ PANSS score } \\
\hline Positive scale & $16(6.72)$ & $7-28$ & & \\
\hline Negative scale & $13.91(5.54)$ & $7-22$ & & \\
\hline Total score & $58.91(17.72)$ & $31-85$ & & \\
\hline
\end{tabular}

\begin{tabular}{|c|c|c|c|c|c|}
\hline & \multicolumn{3}{|c|}{ Talairach coordinates $^{a}$} & \multirow[b]{2}{*}{ Size $^{\mathrm{b}}$} & \multirow[b]{2}{*}{$P$} \\
\hline & $x$ & $y$ & $z$ & & \\
\hline \multicolumn{6}{|l|}{ Control group } \\
\hline Right fusiform gyrus & 32 & -74 & -13 & 17 & 0.006 \\
\hline Right middle occipital gyrus & 36 & -74 & -7 & 3 & 0.006 \\
\hline Right superior temporal gyrus & 43 & 4 & -13 & 14 & 0.006 \\
\hline Right middle temporal gyrus & 51 & 7 & -18 & 10 & 0.006 \\
\hline Right insula & 40 & 4 & -2 & 6 & 0.006 \\
\hline Left amygdala/parahippocampal gyrus & -14 & -7 & -13 & 11 & 0.008 \\
\hline Right amygdala & 25 & -7 & -7 & 9 & 0.006 \\
\hline Right parahippocampal gyrus & 25 & -15 & -24 & 19 & 0.006 \\
\hline \multicolumn{6}{|l|}{ Schizophrenia group } \\
\hline Left angular gyrus & -32 & -56 & 37 & 26 & 0.004 \\
\hline Left cingulate gyrus & -18 & -22 & 42 & 20 & 0.004 \\
\hline Left precuneus & -14 & -56 & 48 & 14 & 0.004 \\
\hline Left medial frontal gyrus & -7 & -15 & 53 & 11 & 0.004 \\
\hline \multicolumn{6}{|l|}{ Control > schizophrenia } \\
\hline Right fusiform gyrus & 25 & -77 & -13 & 19 & 0.004 \\
\hline Left superior temporal gyrus & -47 & -22 & 9 & 13 & 0.0006 \\
\hline Right amygdala & 22 & -7 & -13 & 3 & 0.0007 \\
\hline Right parahippocampal gyrus & 25 & -26 & -7 & 41 & 0.0007 \\
\hline Left inferior frontal gyrus & -51 & -33 & 48 & 37 & 0.0006 \\
\hline Right inferior frontal gyrus & 29 & 15 & -13 & 15 & 0.0007 \\
\hline
\end{tabular}



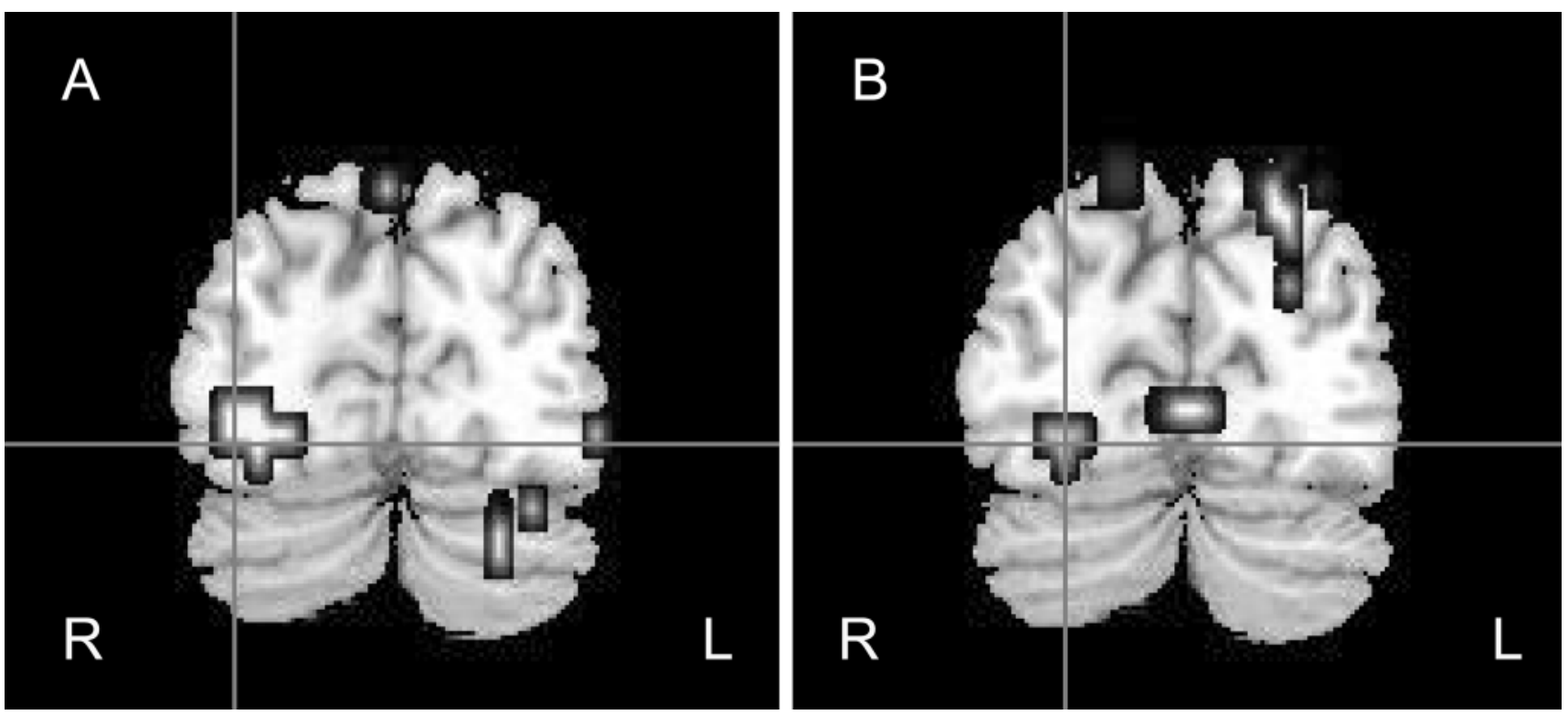

Fig. 1 Coronal view of the brain showing right fusiform gyrus responses to fearful $v$. neutral faces (a) in the control group ( $x=32, y=-74$, $z=-13)$ and (b) the between-group differences, where control participants demonstrated greater activation than the participants with schizophrenia ( $x=25, y=-77, z=-13$ ). $R$, right hemisphere; $L$, left hemisphere.

Patient group: fearful $v$. neutral faces. The processing of fearful faces elicited significantly greater activation within the left medial frontal gyrus, the left angular gyrus, the cingulate gyrus, and left precuneus than did processing of neutral faces (Table 2).

\section{Between-group comparisons}

The whole-brain ANOVA revealed the following between-group significant differences:

Control v. patient groups: activation to fearful faces. Control participants demonstrated significantly greater activation than did the participants with schizophrenia during the processing of fearful faces within the right fusiform gyrus, the left superior temporal gyrus, the bilateral inferior frontal gyri, the right amygdala and right parahippocampal gyrus (Table 2; Figs 1(b), 2(b)).

\section{Correlational analyses}

Correlational analyses in the patient group revealed a significant negative correlation between the PANSS negative sub-scale score and activation within the left superior temporal gyrus during the processing of fearful faces (Pearson $r=-0.84, P=0.001$; Spearman's rho $=-0.74, \quad P=0.01$ ) (Fig. 3). This correlation
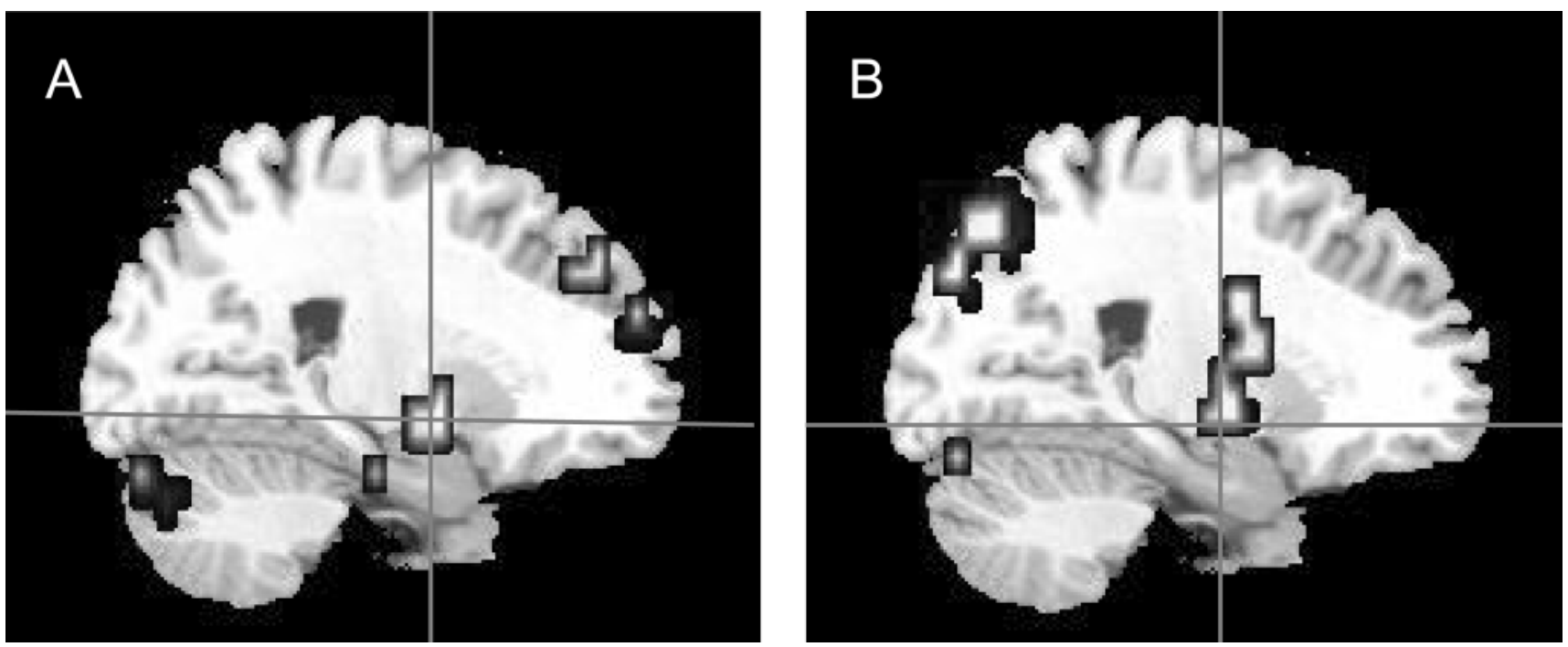

Fig. 2 Sagittal view of the brain showing right amygdala responses to fearful $v$. neutral faces (a) in the control group $(x=25, y=-7, z=-7)$ and (b) the between-group differences, where control participants demonstrated greater activation than participants with schizophrenia $(x=22, y=-7, z=-13)$. 

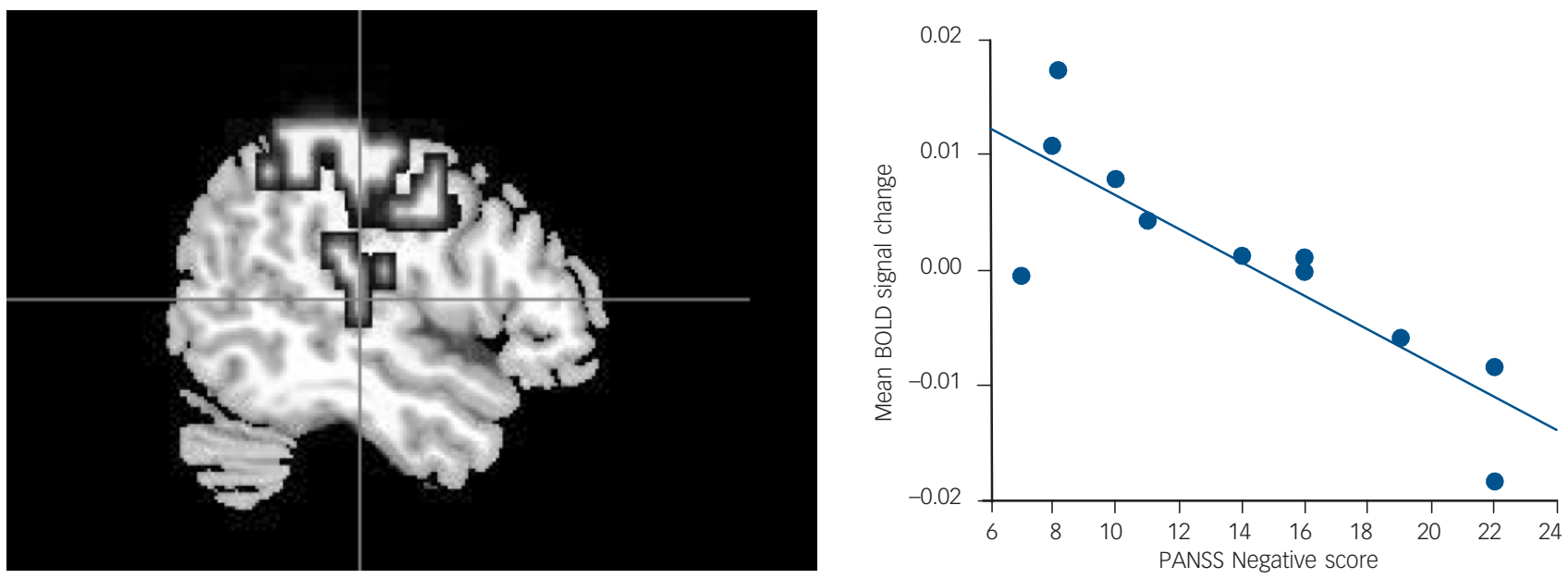

Fig. 3 Sagittal view of the brain showing between-group differences in neural response of left superior temporal gyrus to fearful faces, where control participants demonstrated greater responses than participants with schizophrenia $(x=-47, y=-22, z=9)$. The graph shows the correlation between the Positive and Negative Syndrome Scale (PANSS) negative sub-scale scores and the mean blood oxygen level dependent (BOLD) signal change in the left superior temporal gyrus of participants with schizophrenia (Pearson $r=-0.84, P=0.001$; Spearman's rho $=-0.74, P=0.01$ ).

remained significant after controlling for the patients' IQ $(r=-0.805$, d.f. $=9, P=0.005)$ and gender $(r=-0.847$, d.f. $=9$, $P=0.002)$. No other correlation between measured brain activity and PANSS scores was statistically significant.

\section{Discussion}

Visual processing of facial fear elicited bilateral amygdala activation in healthy participants, with significantly greater activation within the right amygdala compared with the participants with schizophrenia, consistent with earlier reports. ${ }^{15,16}$ This dysfunctional amygdala activation might relate to the fear recognition deficits manifested by patients with schizophrenia. ${ }^{16}$ The amygdala has been postulated to have a key role in modulating the dopaminergic system. ${ }^{17}$ Disruption of dopamine regulation is considered a key factor in the generation of psychotic symptoms by attributing salience and reinforcing aberrant brain circuitry. A previous positron emission tomography (PET) study ${ }^{18}$ demonstrated a positive correlation between the left amygdala response of patients with schizophrenia and the severity of positive symptoms. However, contrary to our prediction, severity of positive symptoms was not associated with a commensurate increase in activation of the amygdala. It is possible that amygdala activation might be linked non-specifically with the illness or medication, rather than the symptom profile. An alternative explanation might be that there is insufficient variation in the positive symptoms of participants with schizophrenia to show a clear correlation, and longitudinal examination of a more symptomatic group of patients could clarify this point.

Healthy participants demonstrated greater activation within the right fusiform gyrus and superior temporal gyrus in response to fearful compared with neutral faces. This finding supports enhancement of extrastriate visual cortices activation by emotionally salient stimuli. ${ }^{5,6}$ In contrast, participants with schizophrenia showed significantly reduced activation within these regions during facial fear processing compared with healthy controls. This is consistent with earlier findings, ${ }^{19-21}$ and may reflect a deficit in the visual processing of facial fear in people with schizophrenia.

Participants with schizophrenia demonstrated a left lateralised reduction in superior temporal gyrus activation, similar to the findings of Johnston et al. ${ }^{22}$ The left lateralised reduction in superior temporal gyrus activation in our study may represent some reversal of the normal right lateralised temporal lobe response to facial fear in people with schizophrenia and may be indicative of impaired function of the left superior temporal gyrus during fear processing in schizophrenia. This might reflect a more general emotional deficit, as an abnormal left lateralised temporal lobe activation has been previously demonstrated in response to emotional prosody. ${ }^{23}$

The cortex surrounding the superior temporal sulcus, along with the fusiform gyrus, is associated with social cognition. Specifically, these regions are involved in the visual perception of socially relevant stimuli. ${ }^{24}$ Findings from neuroimaging, electrophysiological and single-cell recording studies have associated the visual perception of biologically salient motion with the activation of the posterior aspects of the superior temporal sulcus and the adjacent superior temporal gyrus. ${ }^{25,26}$ The perception of the changeable aspects of the faces such as facial emotion, gaze direction and lip movements also elicits activation within the superior temporal sulcus and the adjacent superior temporal gyrus, even in response to implied motion evidenced by static visual stimuli, such as facial expressions. ${ }^{25} \mathrm{~A}$ deficit in biological motion perception may result in deficient social perception, and cognition and social functioning. People with schizophrenia and severe negative symptoms exhibit a pronounced difficulty in the recognition of facial emotions, ${ }^{4}$ particularly facial fear. ${ }^{2,27}$ They also exhibit impairments in social cognition tasks such as theory of mind tasks, also associated with negative symptoms. ${ }^{28-30}$ The social cognition deficits may contribute to the social functioning deficits of the patients with negative symptoms. ${ }^{31}$

The cortical processing of motion-related visual stimuli is impaired in people with schizophrenia and this impairment is more intense in those with severe negative symptoms. ${ }^{9}$ This deficit has been associated with the middle temporal cortical visual area, 
which responds to both scrambled motion and biological motion sequences. The association of activation of the superior temporal cortex, which responds selectively to all aspects of biological motion, with negative symptoms has not been investigated. Consistent with our hypothesis, the severity of negative symptoms of the participants with schizophrenia correlated negatively with the degree of activation attenuation within the left posterior superior temporal gyrus. Our finding indicates an association between impaired extrastriate cortical visual processing of facial fear and negative symptoms in people with schizophrenia. This association may underlie the previously reported pronounced difficulties of patients with high levels of negative symptoms in the recognition of fearful faces.

The neural response within the right fusiform gyrus in the participants with schizophrenia in our study did not correlate with the severity of negative symptoms. This is at odds with an earlier PET study, ${ }^{8}$ which showed decreased glucose metabolic rate in the right fusiform area of patients with predominantly negative symptoms and a negative correlation with the negative symptoms of the patients. The finding of Potkin et $a l^{8}$ was regarded as consistent with the difficulties of the patients with negative symptoms in identifying the emotional content of faces and scenes. The differences in these results may be secondary to the different time frames between eventrelated fMRI and PET, as the occipital cortices are associated with early visual processing.

Deficits in visual tasks in the participants with schizophrenia could be attributed to their attentional deficits, which could lead to lower engagement with the task during the scanning session. However, the findings of our study are not likely to derive from the patients' attentional impairments, since their performance in the gender discrimination task did not differ significantly from the control group's performance.

\section{Methodological considerations}

There are several potential limitations to this study. First is the issue of generalisability of these results from the analysis of a relatively small number of participants. A non-parametric statistical approach to the analysis of the imaging data is preferred, and we have used stringent thresholds for all of our analyses to minimise any type I errors; the significance threshold was set to give less than one false-positive cluster over the whole brain. This would suggest that our findings are relatively robust. Second, participant characteristics - including symptoms and medication in the patient group - may also influence the between-group variability. Ideally, we would have recruited unmedicated patients for this study; however, pragmatically these were difficult to ascertain. Examining for medication effects within the patient group, we found no significant correlation between the medication dose and the blood oxygen dependent level response values within the right amygdala, right fusiform gyrus and left superior temporal gyrus. Similar findings have also been demonstrated in unmedicated patients. ${ }^{21}$ One possibility is that activation differences between patients and controls are secondary to differences in performance. The gender discrimination task we used placed minimal performance demands on the participants, ${ }^{32}$ thereby reducing the possibility that performance differences might confound our results.

In summary, we have confirmed that patients with schizophrenia demonstrated reduced neural responses within extrastriate visual cortices and amygdala during processing of facial fear. A left lateralised reduction of superior temporal gyrus activity correlated with increasing severity of negative symptoms. This association may underlie the previously reported pronounced difficulties of people with schizophrenia and high levels of negative symptoms in the recognition of fearful faces.

\section{Panayiota G. Michalopoulou, MD, CSI Lab, Department of Psychiatry, Institute of Psychiatry, King's College London, UK and Second Department of Psychiatry, Attikon General Hospital, National and Kapodistrian University of Athens Medical School, Athens, Greece; Simon Surguladze, MD, DSc, Section of Neuroscience and Emotion, Department of Psychiatry, Institute of Psychiatry, Kings College London; Lucy A. Morley, MBBS, PhD, MRCPsych, Department of Psychiatry, Institute of Psychiatry, Kings College London; Vincent P. Giampietro, PhD, Brain Image Analysis Unit, Institute of Psychiatry, Kings College London; Robin M. Murray, MD, DSC FRCPSych Department of PSychiatry, Institute of Psychiatry, King's College London; Sukhwinder S. Shergill, MBBS, PhD, MRCPych, CSI Lab, Departme co London; Sukhwinder S. Shergill, MBBS, PhD, MRCPsych, CSI Lab, Department of Psychiatry, Institute of Psychiatry, King's College London, and Wellcome Department of Imaging Neuroscience, Institute of Neurology, University College London, London, UK}

Correspondence: Dr Panayiota G. Michalopoulou, Box PO 67, Institute of Psychiatry, De Crespigny Park, London SE5 8AF, UK. Email: spdppam@iop.kcl.ac.uk

First received 23 Oct 2006, final revision 19 Aug 2007, accepted 25 Sep 2007

\section{Acknowledgements}

S.S.S. was supported by an Advanced Clinical Training Fellowship from the Wellcome Trust and L.A.M. was funded by the Psychiatry Research Trust.

\section{References}

1 Mandall MK, Padney R, Prasad AB. Facial expressions of emotions and schizophrenia: a review. Schizophr Bull 1998; 24: 399-412.

2 Kohler CG, Turner TH, Bilker WB, Brensinger CM, siegel SJ, Kanes SJ, Gur RE, Gur RC. Facial emotion recognition in schizophrenia: intensity effects and error pattern. Am J Psychiatry 2003; 160: 1768-74.

3 Schneider F, Gur RC, Gur RE, Shtasel DL. Emotional processing in schizophrenia: neurobehavioural probes in relation to psychopathology. Schizophr Res 1995; 17: 67-75.

4 Gur RE, Kohler CG, Ragland JD, Siegel SJ, Lesko K, Bilker WB, Gur RC. Flat affect in schizophrenia: relation to emotion processing and neurocognitive measures. Schizophr Bull 2006; 32: 279-87.

5 Morris JS, Friston KJ, Buchel C, Frith CD, Young AW, Calder AJ, Dolan RJ. A neuromodulatory role for the human amygdala in processing emotional facial expressions. Brain 1998; 121: 47-57.

6 Surguladze S, Brammer MJ, Young AW, Andrew C, Travis MJ, Williams SC, Phillips ML. A preferential increase in the extrastriate response to signals of danger. Neuroimage 2003; 19: 1317-28.

7 Fudge JL, Powers JM, Haber SN. Considering the role of amygdala in psychotic illness: a clinicopathologic correlation. I Neuropsychiatry Clin Neurosci 1998; 10: 383-94.

8 Potkin SG, Alva G, Fleming K, Anand R, Keator D, Carreon D, Doo M, Jin $Y$, Wu JC, Fallon JH. A PET study of the pathophysiology of negative symptoms in schizophrenia. Am J Psychiatry 2002; 159: 227-37.

9 Tadin D, Kim J, Doop ML, Gibson C, Lappin JS, Blake R, Park S. Weakened center-surrounded interactions in visual motion processing in schizophrenia. J Neurosci 2006; 26: 11403-12.

10 Annet M. A classification of hand preference by association analysis. $\mathrm{Br} \mathrm{J}$ Psychol 1970; 61: 303-21.

11 American Psychiatric Association. Diagnostic and Statistical Manual of Mental Disorders (4th edn) (DSM-IV). APA, 1994.

12 Kay SR, Fiszbein A, Opler LA. The positive and negative syndrome scale (PANSS) for schizophrenia. Schizophr Bull 1987; 13: 261-76.

13 Nelson HE. The National Adult Reading Test Manual. NFER-Nelson, 1982.

14 Ekman P, Friesen WV. Pictures of Facial Affect. Consulting Psychologists Press, 1976

15 Phillips ML, Williams L, Senior C, Bullmore ET, Brammer MJ, Andrew C, Williams SC, David AS. A differential neural response to threatening and nonthreatening negative facial expressions in paranoid and non-paranoid schizophrenics. Psychiatry Res 1999; 92: 11-31.

16 Gur RE, McGrath C, Chan RM, Schroeder L, Turner T, Turetsky BI, Kohler C, Alsop D, Maldjian J, Ragland JD, Gur RC. An fMRI study of facial emotion processing in patients with schizophrenia. Am J Psychiatry 2002; 159 1992-9.

17 Fudge JL, Emiliano AB. The extended amygdala and the dopamine system: another piece of the dopamine puzzle. J Neuropsychiatry Clin Neurosci 2003; 15: $306-16$ 
18 Taylor SF, Liberzon I, Decker LR, Koeppe RA. A functional anatomic study of emotion in schizophrenia. Schizophr Res 2002; 58: 159-72.

19 Paradiso S, Andreasen NC, Crespo-Facoro B, O'Leary DS, Watkins GL, Boles Ponto LL, Hichwa RD. Emotions in unmedicated patients with schizophrenia during evaluation with positron emission tomography. Am J Psychiatry 2003; 160: $1775-83$.

20 Williams LM, Das P, Harris AW, Liddell BB, Brammer MJ, Olivieri G, Skerrett D, Phillips ML, David AS, Peduto A, Gordon E. Dysregulation of arousal and amygdala-prefrontal systems in paranoid schizophrenia. Am J Psychiatry 2004; 161: 480-89.

21 Taylor SF, Phan KL, Britton JC, Liberzon I. Neural responses to emotional salience in schizophrenia. Neuropsychopharmacology 2005; 30: 984-95.

22 Johnston PJ, Stojanov W, Devir H, Schall U. Functional MRI of facial emotion recognition deficits in schizophrenia and their electrophysiological correlates. Eur J Neurosci 2005; 22: 1221-32.

23 Mitchell RLC, Elliott R, Barry M, Cruttenden A, Woodruff PW. Neural response to emotional prosody in schizophrenia and in bipolar affective disorder. $\mathrm{Br} \mathrm{J}$ Psychiatry 2004; 184: 223-30.

24 Adolphs R. The neurobiology of social cognition. Curr Opin Neurobiol 2001; 11: 231-39.

25 Allison T, Puce A, McCarthy G. Social perception from visual cues: role of the STS region. Trends Cogn Sci 2000; 4: 267-78.
26 Gallagher HL, Frith CD. Functional imaging of 'theory of mind'. Trends Cogn Sci 2003; 7: 77-83.

27 Van't Wout M, Aleman A, Kessels RPC, Cahn W, de Haan EH, Kahn RS Exploring the nature of facial affect processing deficits in schizophrenia. Psychiatry Res 2007; 150: 227-35.

28 Corcoran R, Mercer G, Frith CD. Schizophrenia, symptomatology and social inference: investigating 'theory of mind' in people with schizophrenia. Schizophr Res 1995; 17: 5-13.

29 Frith $C D$, Corcoran R. Exploring 'theory of mind' in people with schizophrenia. Psychol Med 1996; 26: 521-30.

30 Pickup GJ, Frith CD. Theory of mind impairments in schizophrenia: symptomatology, severity and specificity. Psychol Med 2001; 31: 207-20.

31 Addington J, Addington D. Neurocognitive and social functioning in schizophrenia: a 2.5 year follow-up study. Schizophr Bull 2000; 44: 47-56.

32 Phan KL, Wager T, Taylor SF, Liberzon I. Functional neuroanatomy of emotion: a meta-analysis of emotion activation studies in PET and fMRI. Neuroimage 2002; 16: 331-48.

33 Talairach J, Tournoux P. Co-Planar Stereotaxic Atlas of the Human Brain. Thieme, 1988.

\section{Erotic Dreams in Normal Persons [II Sogno Erotico nell'uomo normale]. (Riv. di Psicol., January-February, 1908.) Gualino, L.}

The subject of sexual activity during sleep has been touched on by various psychologists and alienists and studied in detail in a few individual cases. Gualino appears to be the first to investigate it on a larger scale, and bases his paper on the experiences of 100 persons among his acquaintances, doctors, teachers, etc. (apparently all men), to whom he addressed a series of questions. They had all had experience of the phenomenon which Gualino regards as entirely normal.

Gualino finds that erotic dreams, with emissions (whether or not seminal), began somewhat earlier than the period of physical development as ascertained by Marro for youths of the same part of northern Italy. Gualino found that all his casss had had erotic dreams at the age of seventeen; Marro found 8 per cent. of youths still sexually undeveloped at that age, and while sexual development began at thirteen years erotic dreams began at twelve. Their appearance was preceded in most cases for some months by erections. In 37 per cent. of the cases there had been no actual sexual experiences (either masturbation or intercourse); in 23 per cent. there had been masturbation; in the rest some form of sexual content. The dreams are mainly visual, tactual elements coming second, and the dramatis persona is either an unknown woman (27 per cent. cases) or only known by sight (56 per cent.), and in the majority is, at all events in the beginning, an ugly or fantastic figure, becoming more attractive later in life, but never identical with the woman loved during waking life. This, as Gualino points out, accords with the general tendency for the emotions of the day to be latent in sleep. Masturbation only formed the subject of the dream in four cases. The emotional state in the pubertal stage, apart from pleasure, was anxiety (37 per cent.), desire (17 per cent.), fear (14 per cent.). In the adult stage anxiety and fear receded to 7 per cent. and 6 per cent. respectively. Thirty-three of the subjects, as a result of sexual or general disturbances, had had nocturnal emissions without dreams; these were always found exhausting. Normally (in more than 90 per cent.), erotic dreams are the most vivid of all dreams. In no case was there knowledge of any monthly or other cyclic periodicity in the occurrence of the manifestations. In 34 per cent. of cases they tended to occur very soon after sexual intercourse. In numerous cases they were peculiarly frequent (even three in one night) during courtship, when the young man was in the habit of kissing and caressing his betrothed, but ceased after marriage. It was not noted that position in bed or a full bladder exerted any marked influence in the occurrence of erotic dreams; repletion of the seminal vesicles is regarded as the main factor. 